\title{
Sustancias estupefacientes y educación para la salud.
}

\author{
Narcotic substances and health education
}

\section{Sustancias estupefacientes}

Solange Karina Quijije Segovia ${ }^{(1)}$, Msc

Margoth Elizabeth Villegas Chiriboga ${ }^{(2)}$, Msc

Tania Mercedes Alcázar Pichucho ${ }^{(3)}$, Msc

(1) Universidad Estatal del Sur de Manabí, Ecuador. email: solange.quijije@unesum.edu.ec

(2) Universidad Estatal del Sur de Manabí, Ecuador. email: margoth.villegas@unesum.edu.ec

(3) Universidad Estatal del Sur de Manabí, Ecuador. email: tania.alcazar@unesum.edu.ec

Contacto: solange.quijije@unesum.edu.ec

Recibido: 18-02-2020 Aprobado:23-03-2020

\section{Resumen}

El consumo de sustancias estupefacientes por parte de los adolescentes constituye en el mundo actual, un problema a nivel social, educativo y sanitario, que afecta lo individual, familiar, comunitario, laboral y educacional, este fenómeno no respeta estrato social, edad, sexo, se encuentra en forma masiva en nuestra sociedad, creando dependencia física y psíquica en la persona. El objetivo fue determinar la frecuencia de consume de sustancias estupefacientes y su incidencia en el comportamiento biopsicosocial de los estudiantes de la Unidad Educativa Alejo Lascano, tomando una población de 69 estudiantes entre 14 y 17 años. El objeto de estudio fueron los adolescentes con consumo de sustancias estupefacientes. El campo de estudio esta direccionado en la educación para la salud. Para su desarrollo se utilizó la revisión bibliográfica, el estudio es de tipo cualitativo, utilizando metodología descriptiva y observacional, análisis, síntesis, inductivo - deductivo, además de técnicas de encuesta aplicada a los adolescentes. Los resultados evidencian que el consumo de diferentes tipos de estupefacientes los inician por curiosidad a pesar de tener conocimiento sobre las consecuencias de consumirlos. Se concluye que a pesar que la institución brindan charlas educativas y realiza talleres sobre sustancias estupefacientes el problema continua en aumento, los encuestados hicieron conocer que las charlas son muy impactantes pero que no cumplen con su objetivo de ayudar a los jovenes que están con problemas de adicciones, por lo que se deben precisar intervenciones más efectivas para ayudar a los adolescentes con esta problemática.
Palabras clave: Estupefacientes, adolescentes, consumo problemático, promoción de la salud, drogas ilegales

\begin{abstract}
The consumption of narcotic substances by adolescents is a problem in the world today, social, educational and health, which affects the individual, family, community, work and education, this phenomenon does not respect social strata, age, sex, It is found in mass form in our society, creating physical and psychic dependence on the person. The objective was to determine the frequency of drug use and its impact on the biopsychosocial behavior of the students of the Alejo Lascano Educational Unit, taking a population of 69 students between 14 and 17 years old. The object of study was adolescents with drug use. The field of study is addressed in health education. For its development the bibliographic review was used, the study is of qualitative type, using descriptive and observational methodology, analysis, synthesis, inductive - deductive, in addition to survey techniques applied to adolescents. The results show that the consumption of different types of narcotics starts them out of curiosity despite having knowledge about the consequences of consuming them. It is concluded that despite the fact that the institution provides educational talks and workshops on narcotic substances the problem continues to increase, respondents made known that the talks are very impressive but do not meet their goal of helping young people who are with problems of addictions, so more effective interventions must be needed to help adolescents with this problem.
\end{abstract}

Keywords: Narcotic drugs, adolescents, problematic consumption, health promotion, illegal drugs 


\section{Introducción}

El consumo de sustancias estupefacientes, se entiende como un problema complejo de tipo social, en cuanto a la magnitud de personas que se ven afectadas por este; resalta que el consumo de estas sustancias legales e ilegales se incrementa de forma acelerada cada día, afectando los aspectos culturales, históricos, políticos, sociales, geográficos, educativos y económicos de los países, según lo expuesto por (Mendoza \& Vasgas, 2017).

Las sustancias estupefacientes, en sentido general, alteran las características químicas y físicas de los organismos, afectando de manera directa al sistema nervioso central modificando su estructura y modo de funcionar (Berruecos, 2010). Por su parte, Alcívar, Calderón y Jácome (2015), manifiestan que el consumo de estupefacientes puede presentarse de forma experimental, social, regular, intensa y compulsiva, los sujetos podrían cambiar alternativamente entre estos modos y no desarrollar secuencialmente una dependencia. Lo que podría deberse al efecto de la sustancia según (la cantidad, tipo y frecuencia) para generar transformaciones físicoquímicas en el encéfalo, de la misma manera intervienen los aspectos de vulnerabilidad personal y las condiciones socio-familiares en las que se realiza el consumo (Alcívar, Calderón, \& Jácome, 2015).

Una característica común y central ante el consumo de sustancias estupefacientes es la conducta adictiva, es decir, la pérdida de control. La persona con una conducta adictiva no tiene control sobre esa conducta, además de que la misma le produce dependencia, tolerancia, síndrome de abstinencia y una incidencia negativa muy importante en su vida, que va a ser en muchos casos la causa de que acuda en busca de tratamiento o le fuercen a buscarlo. Becoña y Cortés, definierón como elementos característicos de una adicción: 1) el deseo dominante e irresistible de consumir sustancias estupefacientes y obtenerla de cualquier forma; 2) la tendencia a desarrollar el síndrome de tolerancia; 3 ) el desarrollo de la dependencia física - psíquica con presencia de abstinencia por retirada de la sustancia. El mayor problema que tienen las adicciones no son sólo los efectos que producen a corto plazo. El problema está en los efectos que producen a medio y a largo plazo desarrollando enfermedades en los diferentes órganos del cuerpo (Becoña \& Cortés, 2010).

De acuerdo a la Organización Mundial de la Salud se conoce como adolescente a una persona que comprende entre los 10 a 19 años de edad (Organizacion Mundial de la Salud, 2017) es en esta etapa en donde se desarrolla física y mentalmente, e inicia un proceso de aprendizaje, experimentando conductas nuevas, curiosidad hacia lo desconocido, problemas sociales e influencias de personas, que conllevan en la mayoría de los jóvenes al consumo de sustancias ilícitas, actuando sobre el sistema nervioso central provocando la experimentación de nuevas sensaciones.

Uno de los problemas que más afectan a la sociedad es el consumo de sustancias estupefacientes, en los adolescentes que en su mayoría son los más vulnerables a este tipo de adicciones. En la actualidad existen estrategias internacionales, en los que participan instituciones públicas y privadas, estas forman parte del plan nacional de drogas en el que se suman alrededor de 30 países, los cuales se controlan por medio del observatorio europeo de las drogas y la toxicomanía, en los cuales se incluyen las sustancias legales en ciertos países y las sustancias ilegales que también están dentro de ellos, esta es una estrategia que se basa en diferentes tipos de adicciones que se pueden ver en los países (Moreta, Mayorga, León, \& Ilaja, 2017).

Las motivaciones para el consumo de dichas sustancias son varias, en general se describen problemas familiares como: disgregación, disfuncionalidad y problemas de vinculación afectiva (Sajjadi, Jorjoran, Mahboubi, Rafiey, \& Salimi, 2018). De acuerdo con Ventura, las circunstancias específicas que provocan tentación a usar drogas son: estar en lugares donde todos usen drogas, estar con alguien que esté usando drogas, ir a fiestas con los amigos, observar a alguien usar y disfrutar drogas, sentirse extremadamente ansioso(a), estresado(a), y sentirse frustrado(a) porque las cosas no salen como se quiere (Ventura, 2018). Además por la situación social, para ser aceptados y pertenecer a un grupo de amigos, o por factores socio-familiares y a ellos se suma la facilidad para obtenerlas convirtiéndose en un riesgo elevado para la sociedad, ya que la edad de la adolescencia es altamente vulnerable para esta situación, pues de algún modo existe presión que induce al uso de sustancias que pueden ser de cualquier tipo, como drogas legales o ilegales (Avello, Roman, \& Zambrano, 2017).

Informa la Oficina de Naciones Unidas contra la Droga y el Delito (ONUDC), que unos 250 millones de personas, que equivale al $5 \%$ de la población adulta mundial, consumieron drogas por lo menos una vez en el 2015. Aún más inquietante es el hecho de que unos 29,5 millones de esos consumidores, es decir, el $0,6 \%$ de la población adulta mundial, padecen trastornos provocados por el uso de drogas, lo que significa que su adicción a las drogas es perjudicial hasta el punto de que pueden sufrir drogodependencia y necesitar tratamiento (UNODC, 2018).

El análisis de múltiples estudios llevados a cabo en América Latina y que han sido sintetizados por la Organización de los Estados Americanos (OEA), permiten concluir lo siguiente: Colombia ocupa el primer lugar en el subcontinente en lo que a consumo 
temprano de alcohol y uso de tranquilizantes se refiere; Chile tiene el consumo más alto de marihuana; Argentina el de cocaína; y Bolivia parece tener la mayor proporción de consumidores problemáticos, el consumo de heroína ha tenido un incremento muy rápido en pocos años en algunos países, esto particularmente en Brasil, Argentina, Chile y Colombia (OEA, 2013).

Según la Cuarta Encuesta Nacional en Ecuador sobre uso de drogas en estudiantes de 12 a 17 años elaborada por el CONSEP a 514.962 estudiantes encuestados a nivel nacional el 18,3\% declararon hacer uso de estas sustancias durante el último año y que son conseguidas a través de un amigo, el 18,0\% señala que las adquiere a través de un proveedor, el $16,1 \%$ las compran en los alrededores de la institución educativa (CONSEP, 2012), (MSP, 2018).

A partir de lo expuesto anteriormente y partiendo de entender la educación según Quesada como un proceso optimizador y de integración, y la salud como bienestar físico psíquico y social, podemos definir de forma provisional la educación para la salud como «un proceso de educación permanente que se inicia en los primeros años de la infancia orientado hacia el conocimiento de sí mismo en todas sus dimensiones tanto individuales como sociales, y también del ambiente que le rodea en su doble dimensión, ecológica y social, con objeto de poder tener una vida sana y participar en la salud colectiva» (Peñaranda, López, \& Molina, 2017).

Parece claro que la educación para la salud tiene como principal finalidad la mejora cualitativa de la salud humana, centrándose no en conductas aisladas, ya que éstas suponen realidades segmentadas del comportamiento y por ende complejas para su modificación, sino en un entramado de comportamientos que forman los estilos de vida; por otra parte, el hombre en el uso de su libertad no es un ser determinado, aunque sí condicionado por una la educación para la salud reto de nuestro tiempo, guiados por una serie de factores ambientales y también por sus propios hábitos de conducta, por lo que el esfuerzo y la lucha personal deben orientarse no sólo hacia el cambio de aquellos factores nocivos del contexto, sino también a sus propios hábitos y tendencias (Carrillo, 2018).

A pesar de los esfuerzos del gobierno por tratar de disminuir el consumo de sustancias estupefacientes en las instituciones de educación, trabajando con programas educativos a partir de los departamentos de Consejería Estudiantil (DECE) en el cantón Jipijapa este problema de salud pública continua en aumento, se puede evidenciar en el alto consumo de sustancias estupefacientes legales e ilegales, aunque no existen datos estadísticos en los que se pueda conocer el impacto que tienen estas sustancias en el cantón, la afluencia de adolescentes atendidos tanto en el centro de salud como en el hospital son altos.

Al analizar la evolución histórica se visualizan cifras alarmantes en el incremento del uso de sustancias ilícitas en edades cada vez más tempranas, y por los factores de riesgo que se presentan desde los colegios que atienden población adolescente, es necesario implementar este tipo de proyecto en atención a la salud de los adolescentes en los que haya participación directa de prestadores de servicios social, intervención de enfermería en los centros estudiantiles, tanto para la detección oportuna y prevención de sustancias psicoactivas; como en el tratamiento y rehabilitación de jóvenes con adicciones. De esta manera se puede identificar que la disciplina de enfermería tiene poca o nula participación en intervenciones específicas para la atención y cuidado de esta población vulnerable.

En la investigación el problema científico encontrado son los adolescentes con consumo de sustancias estupefacientes, se estableció como objeto de estudio el consumo de sustancias estupefacientes, siendo motivo de investigación el comportamiento de los adolescentes bajo los efectos de las mismas, el campo de la investigación esta direccionado en educación para la salud, teniendo como objetivo general determinar la frecuencia del consumo de sustancias psicoactivas y su incidencia en el comportamiento biopsicosocial de los estudiantes de Primero de Bachillerato de la Unidad Educativa Alejo Lascano.

\section{Materiales y métodos}

Se realizó un estudio cualitativo, utilizando metodología descriptiva y observacional. Para el cumplimiento de esta metodología se utilizaron métodos teóricos como: el análisis-síntesis, inductivo-deductivo para la redacción del informe escrito, marco teórico, el análisis de los resultados; los métodos empíricos utilizados para diagnosticar el problema de los adolescentes con consumo de sustancias psicoactivas fueron las encuestas realizadas. Se trabajó con una muestra de 69 estudiantes, en edades comprendidas entre 14 a 17 años. Todos los estudiantes que participaron en el estudio firmaron el asentimiento informado y sus padres el consentimiento informado. Tanto las encuestas como las charlas educativas fueron realizadas en el plantel educativo. Los aspectos éticos por parte de la investigadora fueron manejados con confidencialidad.

\section{Resultados}

En la tabla 1, se observa que a partir de los resultados del estudio se pudo conocer las sustancias que mayormente predominan en el consumo y que a pesar 
de conocer los riesgos de este consumo lo hacen con mucha frecuencia.

\begin{tabular}{|l|l|l|}
\hline Alternativas & F & $\%$ \\
\hline Mucho & 50 & $72 \%$ \\
\hline Poco & 19 & $28 \%$ \\
\hline Nada & 0 & $0 \%$ \\
\hline
\end{tabular}

Tabla 1. ¿Qué nivel de conocimientos tiene sobre las sustancias estupefacientes?

Fuente: Estudiantes de bachillerato de la Unidad Educativa Alejo Lascano

De acuerdo a la tabla 2, se puede identificar que los estudiantes tienen un satisfactorio nivel de conocimiento de la existencia de las sustancias estupefacientes, sin embargo el consumo refleja altas tasas.

En la tabla 3 se aprecia que los estudiantes reciben información sobre los efectos de las drogas de abuso para la salud; en mayor porcentaje la adquieren del internet (58\%), dentro de las instituciones educativas (14\%) y medios de comunicación (13\%). Por lo cual deberían de no consumirlas, sin embargo los resultados que se reflejan son alarmantes, ya que al tener conocimiento el consumo de estas sustancias es mayor. (tabla 3)

\begin{tabular}{|l|l|l|}
\hline Alternativas & F & $\%$ \\
\hline Alternativas & F & $\%$ \\
\hline Marihuana & 20 & 29 \\
\hline Crippy & 4 & 6 \\
\hline "H" & 5 & 7 \\
\hline Cocaína & 4 & 6 \\
\hline Heroína & 2 & 3 \\
\hline Tabaco y alcohol & 38 & 55 \\
\hline Otras & 0 & 0 \\
\hline
\end{tabular}

Tabla 2. ¿De las siguientes sustancias estupefacientes cuales conoces usted?

Fuente: Estudiantes de bachillerato de la Unidad Educativa Alejo Lascano

\begin{tabular}{|l|l|l|}
\hline Alternativas & $\mathrm{F}$ & $\%$ \\
\hline Internet & 40 & 58 \\
\hline Instituciones de salud & 6 & 9 \\
\hline $\begin{array}{l}\text { Familiares, amigos, } \\
\text { conocidos }\end{array}$ & 4 & 6 \\
\hline $\begin{array}{l}\text { Instituciones } \\
\text { educativas }\end{array}$ & 10 & 14 \\
\hline $\begin{array}{l}\text { Medios de } \\
\text { comunicación }\end{array}$ & 9 & 13 \\
\hline TOTAL & 69 & 100 \\
\hline
\end{tabular}

Tabla 3. ¿De dónde proviene la información que conoce sobre sustancias estupefacientes?

Fuente: Estudiantes de bachillerato de la Unidad Educativa Alejo Lascano
Se evidencia que los estudiantes tiene conocimiento de las sustancias estupefacientes en especial la marihuana, tabaco, alcohol y la denominada droga "H", sin embargo conociendo los daños psicosociales que producen las consumen. (tabla 4)

\begin{tabular}{|l|l|l|}
\hline Alternativas & F & $\%$ \\
\hline Marihuana & 15 & 22 \\
\hline Crippy & 8 & 12 \\
\hline "H" & 12 & 17 \\
\hline Cocaína & 10 & 14 \\
\hline Heroína & 4 & 6 \\
\hline Tabaco y alcohol & 20 & 29 \\
\hline Otras & 0 & 0 \\
\hline TOTAL & 69 & 100 \\
\hline
\end{tabular}

Tabla 4. ¿Ha consumido alguna de las siguientes sustancias estupefacientes?

Fuente: Estudiantes de bachillerato de la Unidad Educativa Alejo Lascano

En la tabla 5, se puede apreciar que una vez que tienen conocimiento de estas sustancias adictivas también las consumen en su mayoría. Lo que refleja que hacen falta programas de intervención mucho más específicas, encaminadas a disminuir el consumo de estas sustancias ilícitas.

Se evidencia además que los estudiantes consumen diariamente sustancias estupefacientes, siendo este su mayor porcentaje, seguido de los que la consumen muy rara vez. El consumo diario se podría interpretar como adicción, siendo esta la imperiosa necesidad que tiene una persona por consumir sustancias como la marihuana, cocaína, inhalantes, tranquilizantes, alucinógenos, etc. Esta necesidad no desaparece aunque la persona padezca consecuencias negativas producidas al momento o después del consumo.

\begin{tabular}{|l|l|l|}
\hline Alternativas & F & $\%$ \\
\hline Todos los días & 25 & 54 \\
\hline Una vez a la semana & 6 & 9 \\
\hline $\begin{array}{l}\text { 2 a 3 veces por } \\
\text { semana }\end{array}$ & 4 & 6 \\
\hline Cada 15 días & 5 & 7 \\
\hline Una vez al mes & 7 & 10 \\
\hline Muy rara vez & 22 & 14 \\
\hline Nunca & 0 & 0 \\
\hline TOTAL & 69 & 100 \\
\hline
\end{tabular}

Tabla 5. ¿En caso de que consuma alguna sustancia estupefacientes, con qué frecuencia lo realiza?

Fuente: Estudiantes de bachillerato de la Unidad Educativa Alejo Lascano

En relación a los factores de riesgo del consumo de estupefacientes, la tabla 6 muetra que en su mayor 
porcentaje está relacionada a la curiosidad con el $44 \%$, seguida por la influencia de grupos de amigos con un $29 \%$. Estas características personales, sociales y familiares permitirían predecir el desarrollo de la conducta de consumo de drogas y situarían a la persona en una posición de vulnerabilidad hacia ese tipo de comportamiento. Más aun cuando conocemos que la adolescencia representa una etapa de cambios y aprendizaje que puede despertar la curiosidad de los jóvenes y el deseo de experimentar qué se siente estando bajo los efectos de las drogas.

\begin{tabular}{|l|l|l|}
\hline Alternativas & $\mathrm{F}$ & $\%$ \\
\hline Curiosidad. & 30 & 44 \\
\hline $\begin{array}{l}\text { Influencia del grupo de } \\
\text { amigos }\end{array}$ & 20 & 29 \\
\hline Problemas familiares & 10 & 14 \\
\hline Problemas psicológicos & 0 & 0 \\
\hline Problemas personales & 9 & 13 \\
\hline Otros & 0 & 0 \\
\hline TOTAL & 69 & 100 \\
\hline
\end{tabular}

Tabla 6. ¿Qué factor de riesgo atribuye al consumo de sustancias estupefacientes?

Fuente: Estudiantes de bachillerato de la Unidad Educativa Alejo Lascano

La tabla 7 muestra las fuentes de adquisicióm de los estupefacientes por parte de los encuestados. En esta tabla se puede apreciar que las sustancias estupefacientes son adquiridas por sus propios medios o lo comparten con los amigos del colegio y del barrio donde residen, por lo que es preocupante la accesibilidad con la que consiguen las sustancias estupefacientes, sobre todo cuando son compartidas entre amigos.

\begin{tabular}{|l|l|l|}
\hline Alternativas & F & $\%$ \\
\hline Por sus propios medios & 30 & 44 \\
\hline Piden a los amigos & 20 & 29 \\
\hline Familiares les proven & 3 & 4 \\
\hline $\begin{array}{l}\text { Consigue fácilmente en } \\
\text { su barrio }\end{array}$ & 10 & 14 \\
\hline $\begin{array}{l}\text { Compañeros del colegio } \\
\text { la proveen }\end{array}$ & 6 & 9 \\
\hline Por sus propios medios & 0 & 0 \\
\hline TOTAL & 69 & 100 \\
\hline
\end{tabular}

Tabla 7. ¿Cómo hacen los adolescentes para adquirir sustancias estupefacientes?

Fuente: Estudiantes de bachillerato de la Unidad Educativa Alejo Lascano

A pesar de conocer las consecuencias de estas sustancias adictivas el $72 \%$ de los jóvenes estudiantes la consumen, (tabla 8). Lo que refleja la imperiosa necesidad de intervención multidisciplinar para contribuir a disminuir las tazas de consumo en los estudiantes y jóvenes en general.

\begin{tabular}{|l|l|l|}
\hline Alternativas & F & $\%$ \\
\hline $\mathrm{Si}$ & 50 & 72 \\
\hline No & 19 & 28 \\
\hline TOTAL & 69 & 100 \\
\hline
\end{tabular}

Tabla 8. ¿Conoce usted las consecuencias del consumo de sustancias estupefacientes?

Fuente: Estudiantes de bachillerato de la Unidad Educativa Alejo Lascano

Es preocupante que la Unidad Educativa no brinde el apoyo suficiente para superar el problema de consumo de estupefacientes, las pocas veces que brindan charlas educativas en la Institución no son suficientes para concientizar a los adolescentes sobre la problemática existente. (Tabla 9)

\begin{tabular}{|l|l|l|}
\hline Alternativas & F & $\%$ \\
\hline Siempre & 14 & 20 \\
\hline A veces & 55 & 80 \\
\hline Nunca & 0 & 0 \\
\hline Total & 69 & 100 \\
\hline
\end{tabular}

Tabla 9. ¿En la institución donde estudia le brindan charlas educativas para orientarlos sobre el consumo de sustancias estupefacientes?

Fuente: Estudiantes de bachillerato de la Unidad Educativa Alejo Lascano

\section{Discusión}

En un estudio realizado en Argentina por Mejía y colaboradores sobre los factores predictores del consumo de sustancias en los adolescentes, explora los factores que predicen el consumo de tabaco, alcohol y otras sustancias en adolescentes Argentinos están asociados con los resultados antes mencionados los factores que muestran mayor asociación con el inicio del consumo de tabaco y alcohol son los hechos de tener mejores amigos fumadores o bebedores (Mejía, y otros, 2019).

En México y Salvador en un estudio realizado por el MINED Ministerio de Educación, se observó que el consumo de tabaco y alcohol se ha reducido pero en forma muy leve, sin embargo el consumo de marihuana se ha incrementado tres veces con relación a años anteriores. Continuando con el análisis, se identifica que la mayoría de los estudiantes que han consumido una psicoactivo lo ha hecho por curiosidad, es decir, son consumidores experimentales (Muñoz, 2014).

En una investigación sobre los factores de riesgo social y consumo de sustancias psicoactivas de 
Andrea Monsalve Valencia, Isabel Cristina Vasco, resaltar que la mayoría de los estudiantes participantes tienen amigos que consumen SPA, además, el $75 \%$ admite que alguna vez sus amigos le han ofrecido consumir SPA; estos resultados son coherentes con el estudio de Caravaca (2015), quienes indican que el $98,5 \%$ de sus participantes reportaron tener por lo menos un amigo que consumía drogas, y que, uno de los factores que más tiene influencia sobre el consuno de SPA en los jóvenes es la influencia del grupo de pares.

\section{Conclusiones}

Las sustancias estupefacientes llamadas comúnmente drogas son sustancias químicas que afectan las funciones del sistema nervioso central es decir al cerebro y la medula espinal, que al ser tomadas pueden modificar la conciencia, el estado de ánimo, o los procesos de pensamiento de un individuo.

El consumo de alcohol y de otras drogas es un problema frecuente principalmente en la adolescencia, etapa del desarrollo donde se forma la identidad individual y se produce la preparación hacia los diferentes roles sociales e individuales, por lo que es fácil entender como en este periodo es frecuente que los adolescentes experimenten con una amplia gama de actitudes y comportamientos entre los que se incluye el consumo de sustancias psicoactivas.

Los factores que determinan que se desarrolle un problema de abuso/dependencia son de naturaleza multifactorial, se han identificado diferentes factores de riesgo que incluyen factores individuales, de grupo de amigos, familiares y de la comunidad, así como influencias genéticas y ambientales. De esta manera la vulnerabilidad genética de un individuo se vería incrementada en presencia de los otros factores de riesgo predisponiendo así al sujeto al consumo y posteriormente al abuso o dependencia de una determinada sustancia

Aunque el consumo de sustancia no es suficiente para llegar a presentar un abuso o dependencia, un número importante de estos adolescentes va a llegar a reunir los criterios para el diagnóstico de trastorno por abuso de sustancia. La Educación para la Salud es indiscutiblemente uno de los recursos más poderosos para poder concienciar a la población informando a los jóvenes sobre las drogas, de cómo potenciar hábitos de vida saludable y habilidades sociales, así como proponer actividades de ocio y otras alternativas.

\section{Bibliografía}

Alcívar, C., Calderón, J., \& Jácome, N. (2015). El uso de las drogas afecta a la sociedad o "contribuye al turismo"? Y sus riesgos en los jóvenes y adolescentes. Revista contribuciones a las ciencias sociales(27).

Avello, S., Roman, M., \& Zambrano, C. (2017). Intervención socio-comunitaria en programas de rehabilitación psicosocial: Un estudio de caso en dos equipos del sur de Chile. Psicoperspectivas, 16(1), 19-30.

Becoña, E., \& Cortés, M. (2010). Manual de adicciones para psicólogos especialistas en psicología clínica en formación. doi:I.S.B.N 978-84-933094-3-5

Berruecos, L. (2010). Drogadicción, farmacodependencia y drogodependencia: definiciones, confusiones y aclaraciones. Cuicuilco, 17(49).

Carrillo, S. (2018). Habilidades para la vida como elementos en la promoción y educación para la salud en los adolescentes y jóvenes. Archivos Venezolanos de Farmacología y Terapéutica, 37(5), 1-6.

CONSEP. (2012). Cuarta encuesta nacional sobre uso de drogas en estudiantes de 12 a 17 años. Resumen ejecutivo. Quito: Observatorio Nacional de Drogas.

Mejía, R., Morello, P., Pérez, A., Peña, L., Arillo, E., Barrientos, I., . . S Sargent, J. (2019). Movies promote tobacco use amongst adolescents: The need for policies to prevent this phenomenon. Rev Asoc Med Argent, 131(1), 24-31.

Mendoza, Y., \& Vasgas, K. (2017). Factores psicosociacles asociados al consumo y adicción a sustancias psicoactivas. Revista electrónica de psicología Iztacala, 20(1), 129.

Moreta, R., Mayorga, M., León, L., \& Ilaja, B. (Octubre de 2017). Consumo de suatncias legales, ilegales y fármacos en adolescentes y factores de riesgo asociados a la exposición reciente. Health and Addictions salud y drogas, 18(1), 39-50.

MSP. (2018). Ministerio de Salud Pública. Plan Nacional de prevención integral y control del fenómeno social economico de las drogas.

OEA. (2013). El problema de Drogas en las Américas: Alternativas Legales y Regulatorias. Organización de los Estados Americanos. 
Organizacion Mundial de la Salud. (2017). salud de la madre, el recien nacido, del niño y el adolescente. ORGANIZACION MUNDIAL DE LA SALUD.

Peñaranda, F., López, J., \& Molina, D. (2017). La educación para la salud en la salud pública: un análisis pedagógico. 22(1), 123-133. doi:0.17151/hpsal.2017.22.1.10

Quesada, R. P. (2013). Promocion y Educacion para la Salud. España: Diaz de Santos.

Sajjadi, H., Jorjoran, S., Mahboubi, H., Rafiey, Y., \& Salimi. (2018, April). Effect of socioeconomic status, family smoking and mental health through social network on the substance use potential in adolescents: a mediation analysis. Public Health. 157, 1419. doi:https://doi.org/10.1016/j.puhe.2018.01.00 4

UNODC. (2018). Informe Mundial sobre las drogas 2018. Resumen, conclusiones y consecuencias en materia de políticas. Oficina de las Naciones Unidas contra la Drgas y el Delito. UNODC Research, 24.

Ventura, J. (Octubre de 2018). Drug initiation and abuse during adolescence: a narrative review / A iniciação e abuso de drogas na adolescência: revisão narrativa. Revista de pesquisa: Cuidado é Fundamental Online, 10(4), 1169-1175.

doi:http://dx.doi.org/10.9789/21755361.2018.v10i4.1169-1175.

Yulis, P. B., Arely, F. H., Amalvis, A. A., Angelica, M. D., \& Zoraya Torres, B. (2015). causas y consecuencias del consumo de psicoactivas en adolescentes con ambientes de vulnearbilibad familiar y contextos sociales conflitivos . tesis , universidad nacional abierta y a distancias UNAD, escuela de ciencias sociales arte y humanidades, santa martha . 